\title{
The use of chemical occurrence and concentration data in exposure assessments of additive intakes in the diets of Irish children as part of a pan-European chemical exposure surveillance system
}

\author{
A. M. Connolly, A. P. Hearty and M. J. Gibney (on Behalf Of The Facet Project) \\ UCD Institute of Food and Health, University College Dublin, Belfield, Dublin 4, Republic of Ireland
}

Effective risk assessment strategies are necessary when monitoring the presence of chemicals in contemporary food supplies, an essential step in these strategies being exposure assessments. Exposure assessments require a certain level of data: (1) concentration levels of the chemical substance, and (2) total intake of foods that contain the chemical ${ }^{(1)}$. Concentration levels can be actual or reflect maximum permitted levels (MPL's) as per the relevant legislation. In the absence of actual occurrence data, it must be assumed that if an additive can legally be present in a food, it always will be. The use of chemical occurrence and concentration data refines exposure assessments to that particular chemical e.g. a food additive.

FACET ${ }^{(2)}$ is a $7^{\text {th }}$ Framework EU funded project aiming to create a food chemical exposure surveillance system, with data from 8 EU countries (including Ireland). An element of this project is obtaining additive occurrence data. A number of target food-groups were developed (n12) and target additives identified for deeper analysis (n32), with specified typologies of foods purchased per food-group to investigate additive occurrence. A second element of this project is the collection of additive concentration data per food-group. Concentration ranges were developed using minimum and maximum concentration values collected from a number of food industries and manufacturers via a member of the FACET project.

Using both the additive occurrence and concentration data collected through FACET, four exposure models were developed. (1) assuming $100 \%$ additive occurrence per food-group with the MPL, (2) assuming $100 \%$ additive occurrence per food-group with true additive concentration data, (3) additive occurrence data with the MPL, and (4), additive occurrence data with true additive concentration data. Dietary data from The National Children's Food Consumption Survey (NCFS) (2003-2004) ${ }^{(3)}$ was utilised with the four exposure models for this research to examine exposure levels of Irish children to three of the target additives. The aim of this study is to highlight the usefulness of incorporating both occurrence and concentration data into an exposure assessment to refine results, thus increasing accuracy.

\begin{tabular}{|c|c|c|c|c|c|c|}
\hline & \multicolumn{2}{|c|}{ Quinoline Yellow ADI (0-0.5) } & \multicolumn{2}{|c|}{ Sunset Yellow ADI $(0-1.0)$} & \multicolumn{2}{|c|}{ Ponceau 4R ADI (0-0.7) } \\
\hline & Mean (SD) & P97.5 & Mean (SD) & P97.5 & Mean (SD) & P97.5 \\
\hline Model 1 & $2.14(3.44)$ & 4.71 & $1.51(2.52)$ & 3.55 & $1.50(2.50)$ & 3.54 \\
\hline Model 2 & $0.49(0.86)$ & 1.16 & $0.34(0.59)$ & 0.90 & $0.62(1.11)$ & 1.60 \\
\hline Model 3 & $0.03(0.11)$ & 0.22 & $0.02(0.08)$ & 0.15 & $0.008(0.03)$ & 0.081 \\
\hline Model 4 & $0.010(0.04)$ & 0.095 & $0.009(0.03)$ & 0.068 & $0.001(0.001)$ & 0.023 \\
\hline
\end{tabular}

SD: Standard Deviation. ADI: Acceptable Daily Intakes $(\mathrm{mg} / \mathrm{kg} / \mathrm{bw})$

P97.5+: $97.5^{\text {th }}$ percentile.

Exposure results were above Acceptable Daily Intakes (ADI) for the most conservative model (model 1), but fell below ADI's for the remaining three models, highlighting the use of chemical occurrence and concentration data in refining exposure analyses and increasing accuracy.

1. Spanjersberg MQI, Kruizinga AG, Rennen MAJ et al. (2007) Risk assessment and food allergy: the probabilistic model applied to allergens. Food and Toxicology 45, 49

2. FACET (Flavourings, Additives and Contact Materials Exposure Task), EU $7^{\text {th }}$ Framework Project, Project No. 211686. http://www.ucd.ie/facet/

3. Irish Universities Nutrition Alliance (2004) The National Children's Food Survey. http://www.iuna.net/doc/bg_ncfs.pdf 